Recherches en didactique des langues et des cultures

Les cahiers de l'Acedle

17-2 | 2020

Recherches collaboratives en didactique des langues

\title{
Formes et enjeux des usages du français au collège à Madagascar
}

Daphné Bloch

\section{OpenEdition}

Édition électronique

URL : https://journals.openedition.org/rdlc/7877

DOI : $10.4000 /$ rdlc. 7877

ISSN : 1958-5772

Éditeur

ACEDLE

Référence électronique

Daphné Bloch, «Formes et enjeux des usages du français au collège à Madagascar », Recherches en didactique des langues et des cultures [En ligne], 17-2 | 2020, mis en ligne le 27 avril 2020, consulté le 17 octobre 2022. URL : http://journals.openedition.org/rdlc/7877; DOI : https://doi.org/10.4000/rdlc. 7877

Ce document a été généré automatiquement le 17 octobre 2022

\section{c) (1) $९$}

Creative Commons - Attribution - Pas d'Utilisation Commerciale - Pas de Modification 4.0 International - CC BY-NC-ND 4.0

https://creativecommons.org/licenses/by-nc-nd/4.0/ 


\title{
Formes et enjeux des usages du français au collège à Madagascar
}

\author{
Daphné Bloch
}

1 Il est communément admis par les acteurs du système scolaire que le français est le médium exclusif de la transmission des connaissances en milieu scolaire à Madagascar. Toutefois les interactions sur le terrain sont révélatrices d'une hétérogénéité linguistique sous-tendue par la nécessité de transmettre un savoir et de communiquer d'une part, et par les prescriptions officielles d'autre part. A l'instar des travaux de Gumperz (1989) et de l'école fonctionnelle, le sens et les formes des alternances codiques visibles, mais labiles, sont l'objet de notre présente investigation.

2 En étudiant les pratiques linguistiques au sein de classes de collèges malgaches, nous identifierons quelques déterminants de l'alternance codique pour répondre à ces questions: quelles formes prennent les pratiques francophones dans la sphère scolaire? Quels sont les enjeux didactiques et idéologiques des formes de la communication pédagogique? Que nous révèle de la glottopolitique malgache les crispations ressenties par les acteurs du système scolaire? Comment les pratiques langagières et les inégalités scolaires sont-elles liées à Madagascar?

3 Trois points de vue complémentaires vont être mis en perspective: les usages linguistiques déclarés des élèves, les déclarations des enseignants et les observations de classes filmées.

\section{Une recherche ancrée dans une expérience de terrain}

4 Le choix du terrain a été guidé par une intuition née de notre expérience professionnelle à Madagascar : les disparités culturelles et linguistiques observées selon les régions, influeraient sur les formes de la communication pédagogique, ce qui déterminerait parallèlement les dynamiques d'enseignement/apprentissage. Nos enquêtes se sont déroulées à Antananarivo, la capitale de l'ile, et à Majunga, une ville de province située sur la côte Nord-Ouest du pays. Alors qu'Antananarivo présente des caractéristiques ethniques et linguistiques très homogènes, la ville de province se 
distingue par un métissage important. En bref, on parle à Antananarivo la variété Merina du malgache, mise à l'écrit précocement et sur laquelle le malgache officiel s'est fondé. Selon un schéma de diglossie enchâssée, le malgache officiel jouit d'une force symbolique et institutionnelle (langue nationale), fortement supérieure à la variété parlée à Majunga ${ }^{1}$.

5 Nos investigations ont concerné huit établissements publics et privés du secondaire. 1752 collégiens ont répondu à notre questionnaire, concernant leurs attitudes scolaires et linguistiques; nous avons conduit 30 entretiens auprès d'enseignants des établissements témoins et réalisé 13 vidéos de classes.

Bien que le français soit le médium officiel de la transmission des savoirs scolaires, un système d'enseignement bilingue s'est tacitement installé depuis la politique de relance $\mathrm{du}$ français, ayant suivi la période dite de malgachisation. L'examen des différents corpus recueillis nous permet d'une part, de constater qu'il existe une distance importante entre les représentations linguistiques des élèves et des enseignants et les pratiques linguistiques au sein de la classe; et d'autre part, que la définition de la langue d'enseignement, dans le discours des enseignants et dans les pratiques observées, n'est pas nette mais bien au contraire qu'elle appelle à questionnement.

\section{Langue(s) d'enseignement et représentations sociales des langues}

7 Afin d'identifier la langue assurant les fonctions réelles de médium d'enseignement nous nous sommes intéressée à la langue utilisée à l'oral, dans les explications de l'enseignant et dans les interactions verticales (enseignant-collégiens). Nous avons en effet estimé, à la suite de J.-M Lévy Leblond (cité par Motchane, 1990) que la transmission est avant tout un échange de paroles. Toutefois, consciente que le discours écrit caractérise la transmission scolaire, nous ne manquerons pas d'examiner la place des langues dans les pratiques écrites.

8 La notion de représentations linguistiques renvoie, de façon directe ou indirecte, à la manière dont un locuteur - ou un groupe de locuteurs - perçoit et pratique des langues. L.-J. Calvet (1999) distingue les pratiques linguistiques qui correspondent aux modalités de production des énoncés, et les représentations qui correspondent aux perceptions réflexives des locuteurs sur leurs pratiques et sur celles des autres locuteurs.

B. Py évoque quant à lui le rôle déterminant des représentations sociales des langues en didactique ${ }^{2}$, et distingue, au sein des discours associés à des représentations sociales sur le langage, celles s'adressant à des énonciateurs anonymes et celles ancrées dans un contexte discursif. (Py, 2004, p. 13) Comme, la notion de représentations sociales des langues permet de faire dialoguer les projections des locuteurs sur leur(s) langue(s) et leurs productions langagières et autorise ainsi, à la fois une approche descriptive et explicative, c'est donc celle que nous privilégions pour éclairer les déclarations des collégiens, ainsi que les discours des enseignants relatifs à la langue d'enseignement.

\section{Interactions enseignant - élèves : les déclarations des collégiens}

10 Nous avons respectivement questionné enseignants et élèves au sujet de la langue utilisée au cours des interactions verticales. Aux collégiens, nous avons posé deux 
questions complémentaires. Dans un premier temps, nous leur avons demandé quelle était la langue qu'ils préféraient que l'enseignant utilise pour les séquences explicatives. Puis, nous leur avons demandé quelle(s) langue(s) ils parlaient avec leurs enseignants ${ }^{3}$. Ces questions concernaient les langues utilisées à l'oral. Quant aux enseignants, nous leur avons demandé au cours des entretiens, dans quelle(s) langue(s) ils parlaient quand ils faisaient cours, puis nous leur avons demandé leur point de vue concernant la langue préférée des élèves en classe.

11 Les graphiques suivants (Graphiques 1.1 et 1.2$)^{4}$ présentent la répartition des usages linguistiques déclarés des collégiens avec les enseignants.

\section{Graphique 1.1 : Langues que les collégiens déclarent parler avec leur enseignant - comparaison d'Antananarivo et de Majunga}

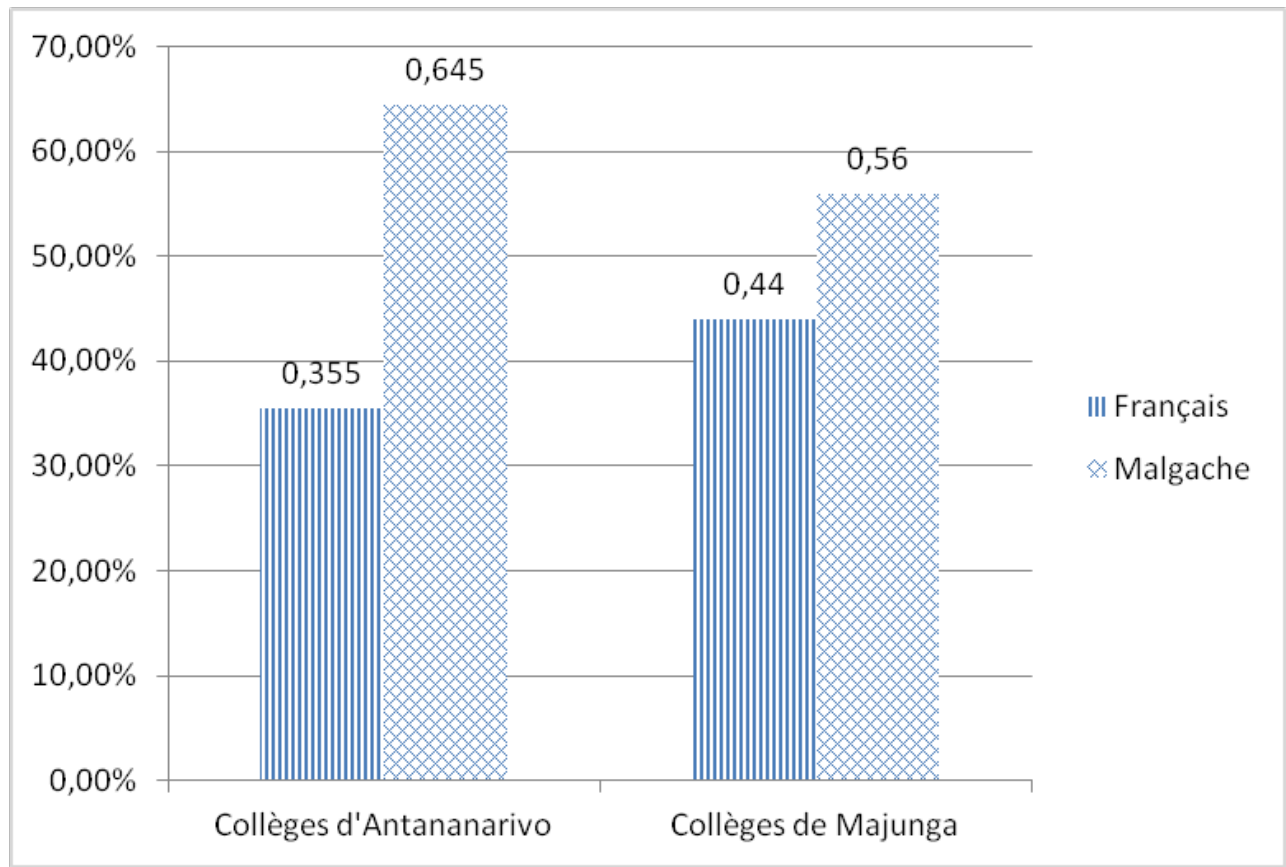

Source : Bloch 2015, enquête réalisée par questionnaires auprès de 1752 collégiens inscrits dans 4 établissements privés et 4 établissements publics.

Le tableau montre un usage déclaré du malgache plus répandu chez les élèves interrogés à Antananarivo qu'à Majunga, ville, qui selon cet indicateur, se révèlerait plus francophone que la capitale. En outre, les déclarations concernant la langue utilisée pour les échanges sont proches dans les deux villes, les chiffres dénotant cependant d'une légère prééminence de la langue malgache, notamment dans les établissements de la capitale. Les observations de classes nous ont appris que les élèves n'étaient qu'exceptionnellement à l'initiative de ce type d'interaction ${ }^{5}$. 
Graphique 1.2: Langues que les collégiens déclarent parler à leur enseignant - comparaison selon la ville et le type d'établissement

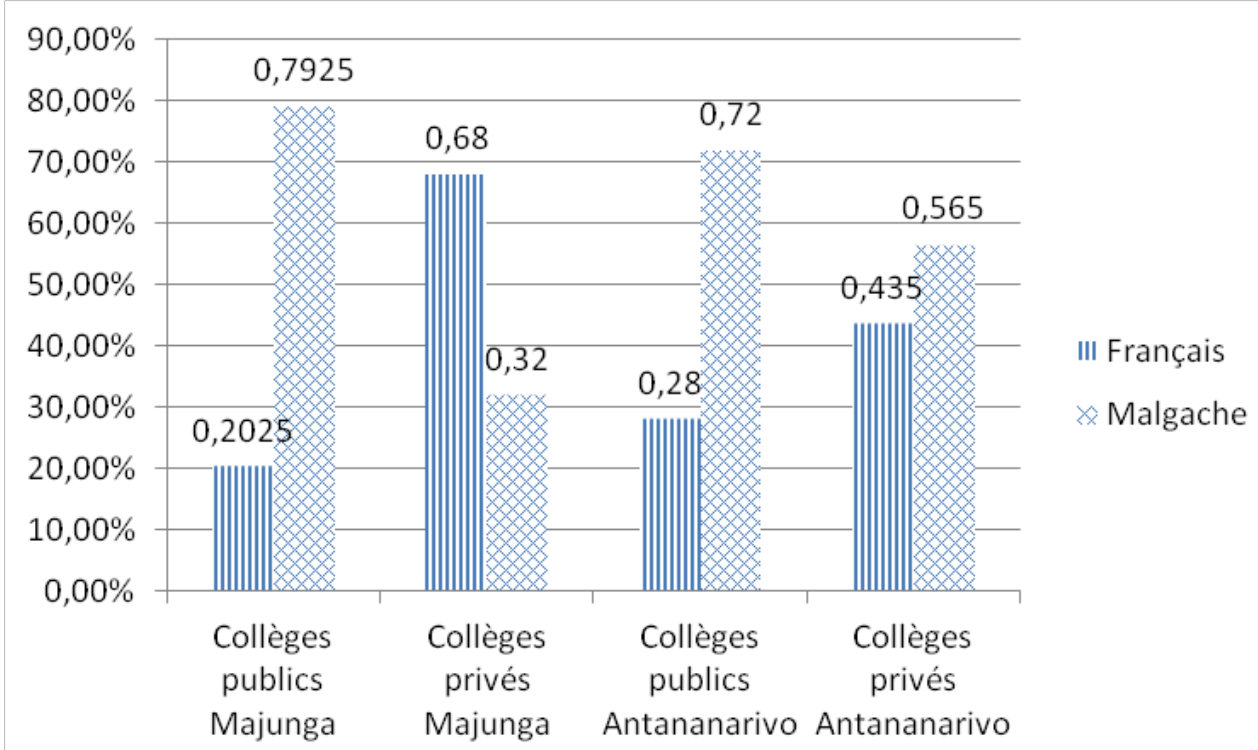

Source : Bloch 2015, enquête réalisée par questionnaires auprès de 1752 collégiens inscrits dans 4 établissements privés et 4 établissements publics.

À Majunga la corrélation entre le type d'établissement et le médium utilisé dans la communication pédagogique est remarquable. D'après les déclarations des élèves, le français domine les échanges enseignants-élèves dans les établissements privés (68\%). Ce sont les seuls collèges où le français domine de façon claire et nette. L'enjeu est à la fois pédagogique et commercial. Ces collèges ont fait de l'usage du français un gage de qualité de l'enseignement, ce qui les rend attractifs ${ }^{6}$. Les résultats du graphique 1.1 montraient des pratiques plus francophones à Majunga que dans la capitale, où l'effet établissement est aussi plus faible que dans la ville de province. Les résultats sont ainsi influencés par les pratiques glottopolitiques des établissements privés de Majunga.

En outre, la proximité du malgache officiel avec la variété parlée dans la capitale, la prédominance et la valorisation de la culture merina jouent un rôle important dans les pratiques linguistiques à l'école: nous constatons que la majorité des échanges enseignants- élèves se font en malgache, et ce quel que soit le type d'établissement. Les usages du français sont plus importants dans les établissements privés, comme on pouvait s'y attendre.

insi, suivant un critère limité aux interactions orales et selon ces déclarations, le malgache assurerait le rôle de langue d'enseignement. Cette fonction du malgache est sans équivoque dans les établissements publics.

La confrontation des réponses obtenues à la question concernant la langue préférée par les élèves pour les explications (graphique 2) ${ }^{7}$ ne traduit qu'une légère préférence pour les explications en malgache selon les données globales, mais nous constatons que la répartition dans les deux villes entre les collèges privés et les collèges publics, dessine des images opposées. 
Graphique 2 : Langues que les collégiens déclarent préférer pour les explications données par leur enseignant - comparaison selon la ville et le type d'établissement

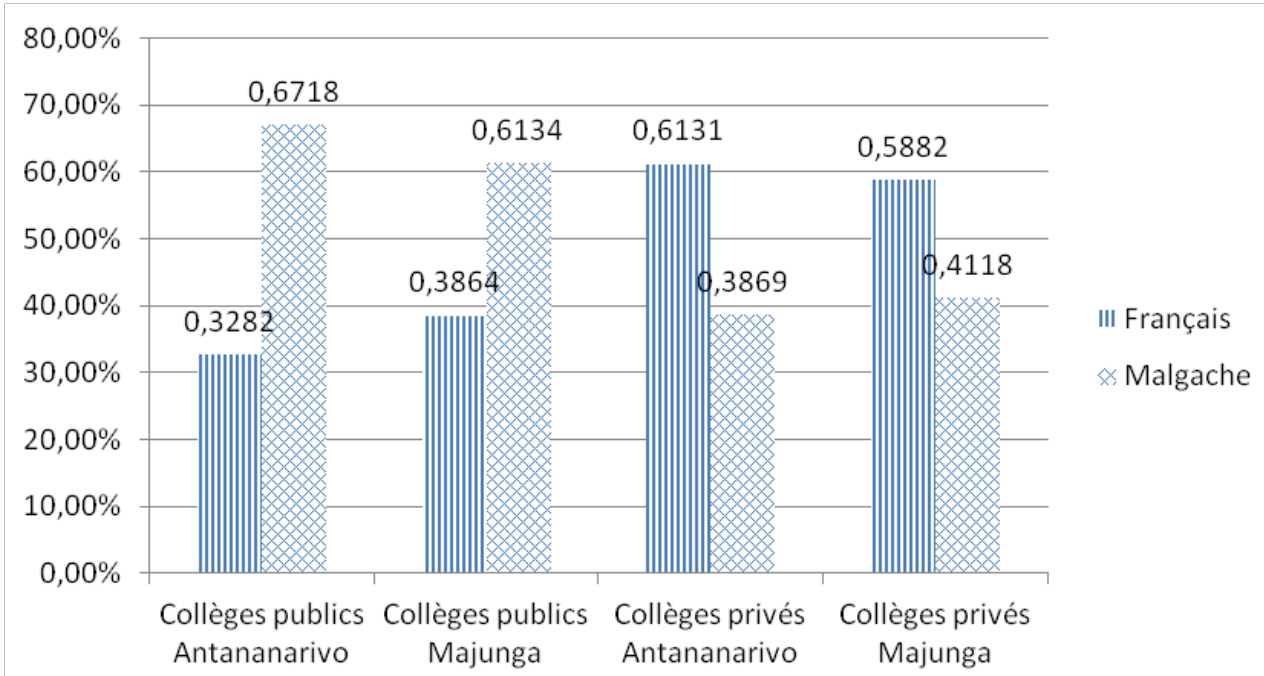

Source : Bloch 2015, enquête réalisée par questionnaires auprès de 1752 collégiens inscrits dans 4 établissements privés et 4 établissements publics.

17 Il est notable que les élèves des collèges publics de Majunga affichent une certaine préférence pour les explications en français par rapport à leurs homologues de la capitale, ce qui peut à nouveau s'expliquer par la distance entre le malgache officiel et la variété majungaise du malgache.

L'analyse statistique des réponses aux questionnaires permet de dessiner deux tendances révélatrices de représentations sociales:

1) Un clivage public / privé très net: les élèves des établissements publics déclarent utiliser davantage le malgache avec leurs enseignants et préférer les explications en malgache, alors que les collégiens scolarisés dans des établissements privés déclarent préférer le français. Nous pouvons supposer que la langue française étant associée à l'excellence scolaire, des enjeux de face ont influencé les réponses des collégiens.

2) Une préférence des collégiens Majungais interrogés pour le français, par rapport aux élèves de la capitale et un usage important du français dans les interactions avec les enseignants dans les établissements privés. Si nous considérons que le malgache employé dans l'enseignement est le malgache officiel (selon les prescriptions ministérielles et les déclarations des enseignants) il est fort probable que cette variété ne soit pas la langue maternelle des élèves de Majunga et que bien au contraire, l'usage du malgache officiel produise sur ces derniers une violence symbolique plus importante que ne provoque l'usage du français (Bourdieu, 1977). La glottopolitique des établissements privés de Majunga pourrait alors se comprendre en réaction face au malgache officiel.

19 Voici une explication très adoucie de la prédilection des élites provinciales pour l'enseignement en français.

Les provinces côtières ( 60 \% de la population) n'ont pas eu la même histoire que les Hauts-Plateaux. Même si elles comprennent très bien la langue officielle, et de mieux en mieux $d u$ fait des contacts, elles ne se sentent pas encore sentimentalement attachées à cette langue «merina ». L'élite de ces provinces hésite donc entre trois politiques linguistiques: promouvoir les dialectes, promouvoir le malgache officiel, promouvoir le français. C'est ce dernier choix 
qu'elle fait en ce moment dans l'enseignement. (Dumont, Rakotozanany \&

Ratsimbazafy, 1995, p. 51) origines remontent aux circonstances de la transcription du malgache en alphabet latin (Raison Jourde, 1991, p. 530) ${ }^{8}$ et à l'influence du purisme français, et qui se manifeste davantage dans le discours métalinguistique des enseignants que dans des pratiques linguistiques?. pseudonymes, exerçant dans des établissements très différents et enseignant des matières appartenant à des champs disciplinaires éloignés: deux enseignants de mathématiques exerçant dans des établissements privés et deux enseignantes d'histoire-géographie exerçant dans des établissements publics. Les extraits d'entretiens présentés ci-dessous illustrent deux types d'usages linguistiques contraints, caractéristiques des représentations sociales des langues des locuteurs.

\section{Le malgache : une langue « mal équipée » pour enseigner}

L'argument du défaut lexical qui affecterait le malgache est souvent avancé lorsqu'il s'agit d'expliquer les raisons de l'usage d'une langue exogène en classe. Les enseignants des matières scientifiques seraient les plus touchés par cette soi-disant carence de la langue malgache.

Corpus Antananarivo : extrait entretien $\mathrm{n}^{\circ} 12$ : M. Mamy, 48 ans, professeur de mathématiques au Collège Saint François-Xavier ${ }^{10}$

D100: d'accord ok / euh quand vous êtes en classe vous parlez en quelle langue? /

M101 : en français ! mais quelques fois si euh si si on voit que les élèves nn'ont pas bien compris alors on est OBligé quelquefois de parler en malgache /

D102 : c'est quelquefois? /

M103 : c'est quelquefois hein pas très souvent // tu vois ce que l'on enseigne c'est là il n'y a pas de mathématiques en malgache hein [D : hum hum] c'est en français alors on est obligés de parler en français oui / 

référence: d'une part, M. Roger ne souhaite pas blesser l'enquêtrice française, d'autre 
part, en tant qu'enseignant du privé il souhaite donner une image francophone et francophile de ses élèves.

L'usage est également modulé dans cet énoncé, porteur lui aussi d'une représentation sociale de référence : "enfin c'est surtout peut-être euh; le malgache / ». Ainsi, bien qu'il semble légitime pour des collégiens malgaches de préférer un enseignement dispensé en langue malgache, l'adverbe "peut-être» nous montre que l'usage du malgache en classe, ainsi que l'intérêt affectif et pédagogique qu'il peut présenter, n'est pas entièrement assumé par cet enseignant. Par ailleurs, le discours de M. Roger se caractérise par des processus d'atténuation, conformément aux cadres conceptuels régissant la communication à Madagascar ${ }^{11}$.

\section{L'obstacle du français}

Les extraits suivants font état de pratiques et de points de vue fortement différents de ceux exprimés par les enseignants de mathématiques, et témoignent de représentations sociales de références et d'usages tout aussi distinctes.

\begin{tabular}{|c|c|}
\hline \multicolumn{2}{|c|}{$\begin{array}{l}\text { Corpus Majunga : extrait entretien } n^{\circ} 3 \text { : Mme Chantal, } 52 \text { ans, professeure d'histoire- } \\
\text { géographie au C.E.G }{ }^{12} \mathrm{Ch} \text {. Renel }\end{array}$} \\
\hline $\begin{array}{l}\text { D94 : alors / pendant la classe / } \\
\text { vous parlez en français ou vous } \\
\text { parlez en malgache? / } \\
\text { C95 : en malgache / TOUT! } \\
\text { D96 : tout? }\end{array}$ & \\
\hline $\begin{array}{l}\text { C97: hum hum / fa presque en } \\
\text { malgache izahay amin'ny } \\
\text { explications de la leçon izany } \\
\text { amin'ny malagasy fa ny leçon en } \\
\text { français }\end{array}$ & $\begin{array}{l}\text { enfin presque en } \\
\text { malgache l'explication de } \\
\text { la leçon en malgache mais } \\
\text { la leçon en français }\end{array}$ \\
\hline $\begin{array}{l}\text { D98 : d'accord ok / et est c'que } \\
\text { vous écrivez au tableau en } \\
\text { français ou en malgache? }\end{array}$ & \\
\hline C99 : en français! & \\
\hline D100 : en français d'accord $\S$ & \\
\hline $\begin{array}{l}\text { I101 : tout ce qui est écrit est en } \\
\text { français/ }\end{array}$ & \\
\hline $\begin{array}{l}\text { D102 : et est-c'que les élèves ils } \\
\text { comprennent le français? }\end{array}$ & \\
\hline C103: tsy izy jiaby / & pas tous / \\
\hline
\end{tabular}




\begin{tabular}{|l|l|l|}
\hline $\begin{array}{l}\text { D104 : alors alors pourquoi vous } \\
\text { faites la leçon en français? }\end{array}$ & & \\
\hline C105: mais c'est obliGé ! & & \\
\hline
\end{tabular}

Mme Chantal avance un argument différent: la contrainte pèserait sur l'emploi du français et serait attribuée aux directives ministérielles imposant l'emploi du français dans l'enseignement ${ }^{13}$. La position de cette enseignante est caractéristique de la schizoglossie. (Guerin, 2010). Pour Mme Chantal, la représentation sociale de référence accorde donc la place de médium d'enseignement au français, quoiqu'une contrainte institutionnelle pèse clairement sur cette représentation; mais dans l'usage c'est le malgache qui assure cette fonction.

Des représentations similaires sont présentes dans le discours de Mme Felana, également professeure d'histoire-géographie. L'extrait d'entretien ci-dessous intervient après l'évocation des difficultés rencontrées par les élèves dans la matière.

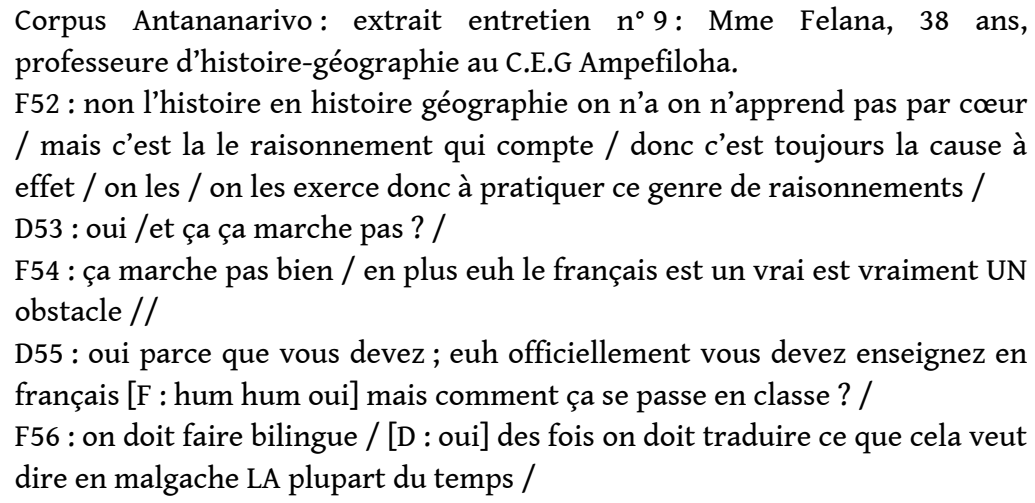

En F65, nous constatons plusieurs signes caractéristiques de représentations sociales : "on doit faire bilingue », il s'agit d'une représentation sociale d'usage. En revanche, l'hésitation qui fait passer Mme Felana de «des fois » à "LA plupart du temps » est caractéristique d'une tension entre ses usages linguistiques et les représentations sociales de références attachées à l'emploi de la langue française. Cependant, le malgache est également pour cette enseignante le médium d'enseignement privilégié dans la communication orale.

\section{Répartition fonctionnelle des langues}

À ce stade, l'analyse nous conduit à penser qu'une seule langue ne suffit à remplir toutes les fonctions nécessaires à l'acte d'enseignement : celles-ci sont partagées entre deux langues et même trois dans le cas où la variété locale du malgache est utilisée selon un schéma de triglossie (Mackey, 2000). Parmi ces fonctions, nous retiendrons celle de donner pour vrai un savoir (Chiss, 2005) ${ }^{14}$; transmettre et expliquer un savoir et évaluer/contrôler la compréhension et les acquisitions des élèves. Les modalités discursives de réalisation de ces fonctions font diversement appel à l'écrit et à l'oral, à l'explicite et à l'affectivité des élèves. 


\section{Les usages du français dans les établissements publics témoins : objectivisation des savoirs, rituels et mémorisation}

Nos observations font état d'un usage quasi-exclusif du français à l'écrit. Cet emploi interroge quant aux raisons conduisant au choix du médium et du canal. En effet, comme l'écrit Razafindratsimba (2009), le malgache pourrait techniquement être employé comme médium à l'écrit. Cependant, qu'il s'agisse d'un héritage colonial désormais associé au désir de modernité et/ou du souvenir de l'échec de la malgachisation de l'enseignement, conjointement à une menace d'isolement linguistique de l'île, c'est le français qui est utilisé selon les déclarations des enseignants pour faire la leçon. Nous retrouvons cette expression dans la totalité des entretiens effectués, la variable matière n'est pas à ce stade pertinente. Le type d'établissement où exercent les enseignants peut toutefois être corrélé avec des modulations du sens porté par cette formule.

Dans les établissements publics, faire la leçon en français correspond à la recopier au tableau à partir d'un livre en français et à la faire copier aux élèves dans leur cahier.

L'usage du français servirait alors ici à objectiver les contenus d'enseignement et à les harmoniser. L'usage du français à l'oral apparait toutefois dans le cadre de formules ritualisées et comme mots-clés à mémoriser au sein d'énoncés explicatifs en malgache. Un exemple intéressant est donné dans l'extrait transcrit ci-dessous :

\begin{tabular}{|c|c|}
\hline \multicolumn{2}{|c|}{$\begin{array}{l}\text { Corpus Antananarivo : vidéo } n^{\circ} 6: \text { un cours d'histoire au C.E.G d'Ampefiloha, classe de } \\
3^{\text {ème }} \\
\text { (C'est le début du cours, les élèves ont leur cahier ouvert.) }\end{array}$} \\
\hline $\begin{array}{l}\text { L'enseignante } 1: \text { quelles sont les } \\
\text { différentes mesures prises par } \\
\text { LES Bolchéviques? ils ont pris } \\
\text { DES Mesures! } \\
\text { (elle écrit "mesures» au } \\
\text { tableau) hum hum? } \\
\text { iza no hamaly? Tsilavina? }\end{array}$ & $\begin{array}{l}\text { qui va } \\
\text { Tsilavina? }\end{array}$ \\
\hline \multicolumn{2}{|l|}{ Tsilavina 2 : décret de paix / } \\
\hline $\begin{array}{l}\text { L'enseignante } 3 \text { : } \\
\text { han han misy zavatra nataony ao } \\
\text { décret de paix / } \\
\text { dia inona no dikan'izany? }\end{array}$ & $\begin{array}{l}\text { voilà ce qu'ils ont fait le } \\
\text { décret de paix / } \\
\text { ça veut dire quoi? }\end{array}$ \\
\hline \multicolumn{2}{|l|}{ Les élèves 4 : arrêtER la GUErre / } \\
\hline $\begin{array}{l}\text { L'enseignante } 5 \text { : } \\
\text { inona no dikan'io ? } \\
\text { (elle désigne un élève) }\end{array}$ & $\begin{array}{l}\text { qu'est-ce que ça veut } \\
\text { dire? }\end{array}$ \\
\hline $\begin{array}{l}\text { L'élève interrogée } 6 \text { : arrêter de } \\
\text { la guerre / }\end{array}$ & \\
\hline
\end{tabular}




\begin{tabular}{|c|c|}
\hline $\begin{array}{l}\text { L'enseignante } 7: \text { arrêTER la } \\
\text { GUErre / } \\
\text { satria ahoana anisan'ny? } \\
\text { jereo tsara tadidintsika taty an! } \\
\text { anisan'ny nahatonga revolution } \\
\text { russe ny conséquence de la guerre / } \\
\text { izany HOE mba hampandroso } \\
\text { ny fiantraikan'ny ady / dia } \\
\text { anisan'ny tao izany an! } \\
\text { nahatanteraka ny ao amin'ny thèse } \\
\text { d'avril aloha izy sa tsy ao izay? } \\
\text { mety tsara aloha izay satria izy hoe } \\
\text { arrêter la? }\end{array}$ & $\begin{array}{l}\text { pourquoi? } \\
\text { regardez bien on se } \\
\text { souvient que ici! } \\
\text { ce qui a causé la } \\
\text { révolution russe c'est une } \\
\text { conséquence de la guerre } \\
\text { / } \\
\text { c'est-à-dire que les } \\
\text { conséquences de la } \\
\text { guerre font avancer le } \\
\text { pays / à part ça! } \\
\text { est-c'qu'il a bien appliqué } \\
\text { la thèse d'avril oui ou } \\
\text { non? } \\
\text { c'était bien car ça à arrêté } \\
\text { la? }\end{array}$ \\
\hline Les élèves 8 : GUERRE! & \\
\hline
\end{tabular}

« Décret de paix » " arrêté la guerre » et encore « la guerre » sont progressivement les seuls termes énoncés en français par les élèves et l'enseignante. Il s'agit de mots-clés à mémoriser, l'usage du français renvoie donc à la fonction de donner pour vrai un savoir. Le malgache prend rapidement une place prépondérante dans la communication pédagogique et assure les fonctions de transmission, d'explication ainsi que d'évaluation orale de la compréhension. Les interactions sont alors poussives et doivent s'appuyer sur l'écrit par la récitation par cœur et/ou la lecture de la leçon copiée dans le cahier. Le malgache s'introduit rapidement et marque le début des véritables interactions enseignant-élèves, sans qu'aucun recours à l'écrit ne soit plus nécessaire. L'extrait suivant illustre le passage d'un médium à l'autre, le français étant abandonné après une tentative de récitation de la leçon :

\begin{tabular}{|c|c|}
\hline \multicolumn{2}{|c|}{$\begin{array}{l}\text { Corpus Majunga: vidéo } \mathrm{n}^{\circ} 10: \text { un cours de physique-chimie au C.E.G d'Amborovy, } \\
\text { classe de } 4^{\text {ème }}\end{array}$} \\
\hline $\begin{array}{l}\text { L'enseignant } \quad 17: \quad \text { un } \\
\text { conduCTEUR? //est un corps qui } \\
\text { laisse passer? }\end{array}$ & \\
\hline $\begin{array}{l}\text { Les élèves } 18 \text { : est un corps qui } \\
\text { laisse passer le courant } \\
\text { électrique / }\end{array}$ & \\
\hline $\begin{array}{l}\text { Une élève } 19 \text { : raha ohatra hoe hafa } \\
\text { ny isolant sy conducteur? }\end{array}$ & $\begin{array}{l}\text { mais quelle est la } \\
\text { différence entre isolant et } \\
\text { conducteur? }\end{array}$ \\
\hline $\begin{array}{l}\text { L'enseignant } 20 \text { : (s'adressant à } \\
\text { l'élève) ka mitovy ny isolant sy } \\
\text { conducteur? }\end{array}$ & $\begin{array}{l}\text { est-ce que c'est pareil } \\
\text { isolant et conducteur? }\end{array}$ \\
\hline
\end{tabular}




\begin{tabular}{|l|l|}
\hline $\begin{array}{l}\text { L'élève } 21: \text { ha ha } \\
\text { fa rehefa hoe samby hafa! }\end{array}$ & $\begin{array}{l}\text { non } \\
\text { c'est différent ! }\end{array}$ \\
\hline $\begin{array}{l}\text { L'enseignant } 22 \text { : (s'adressant à la } \\
\text { classe) } \\
\text { han ka mitovy ve ny isolant sy } \\
\text { conducteur? }\end{array}$ & $\begin{array}{l}\text { hum est-c'que c'est pareil } \\
\text { isolant et conducteur? }\end{array}$ \\
\hline Les élèves 23:TSY MItoVY! & c'est pas pareil! \\
\hline
\end{tabular}

Dès le tour de parole 19, le français n'est plus conservé que sous la forme d'emprunts insérés dans les énoncés en malgache ( ny isolant sy conducteur » aux tours de parole 19, et 22 et "courant» présent deux fois au tour de parole 27). Le passage au malgache permet un déplacement des frontières didactiques et linguistiques entre enseignant et enseignés, une élève allant jusqu'à poser une question à l'enseignant sans même lever la main (tour de parole 19). Il est notable que la prise de parole de chacun, « délivrée » en quelques sortes, de la langue de l'écrit, est plus spontanée en malgache. Si le français est présent à l'écrit et à l'oral au début du cours, il est rapidement abandonné par les enseignants $\mathrm{du}$ public. Le malgache assume donc les fonctions de médium d'enseignement dans les établissements publics témoins.

\section{Les usages du français dans les établissements privés témoins : explications et raisonnement}

Les enseignants des établissements privés s'efforcent quant à eux de donner également des explications en français. Au cours de nos observations nous avons considéré qu'il y avait deux types d'emplois du français à l'oral par les enseignants du privé. Le premier consiste en des explications de règles, et aurait alors pour objectif de permettre aux élèves de raisonner en français. Cet emploi consiste en des interactions verticales en français constatées dans un cours de français à l'ESCA, un des plus prestigieux établissements de la capitale. La leçon portait sur la transformation des phrases actives en phrases passives. La leçon est ici expliquée en français à partir d'exemples d'énoncés, et non pas d'une leçon écrite au tableau. L'enseignante s'assure de la compréhension des élèves et leur pose des questions collectives. Il y a donc un échange oral en français entre elle et la classe. Les réponses orales des élèves sont cependant fortement ritualisées : rompus à l'exercice de l'écoute active, ils finissent en chœur les phrases de leur enseignante, afin de montrer leur attention et leur compréhension. Le rythme et l'accentuation caractérisant les propos de l'enseignante jouent un rôle important pour le repérage et la compréhension des mots-clés par les élèves. La variable matière (ainsi que la variable établissement) est ici en corrélation directe avec l'emploi du français dans les interactions.

Le second type de leçon donnée et expliquée en français, observée dans un établissement privé de Majunga, consiste davantage en l'oralisation par l'enseignant de la leçon écrite au tableau. Toutefois le schéma discursif est proche de celui repéré dans la leçon de français donnée à l'ESCA. 
Corpus Majunga : vidéo $\mathrm{n}^{\circ} 8$ : un cours de mathématiques au collège SaintGabriel, classe de $4^{\text {ème }}$

(L'enseignant a écrit la leçon portant sur les vecteurs en français au tableau. Les élèves ont recopié la leçon.)

L'enseignant 5 : qu'appelle-t-on vecteurs opposés?

Les élèves 6 : on appelle vecTEURS oppoSÉS / des vecTEURS qui ont la même direction la même lonGUEUR mais SENS contrAIRE /

L'enseignant 7 : on appelle vecteurs opposés / DEUX ou plusieurs vecteurs qui ONt la même direCTION la même lonGUEUR MAIS de SENS contraire [les élèves : contraire] on y VA alors (il lit le tableau) caractérisaTION du milieu des segMENTS / propriéTÉ A B I sont trois points du PLAN / I est le milieu de A B et I A plus I B égal / vecTEUR / NUL / pourQUOI I A plus I B égal vecteur nul ? regardez bien la FIgure ( il pointe la figure avec son doigt) I A plus I B que constatez-vous ici? [les élèves : XX] HEIN? I vers A et I vers B / est ce que le le SENS est toujours le MÊME?

Les élèves $8:$ NON /

L'enseignant 9: HEIN? que peut-on dire alors SI si on additionne deux vecteurs de sens

contraire par exEMple d'après la relation de Chasles (il se met à écrire ce qu'il dit) A B vecteur A B plus vecTEUR B A d'après la relation de Chasles ça donne QUOI?

Les élèves 10 : vecTEUR A /

L'enseignant $11:$ A d'accord ! qu'appelle-t-on vecteur $\underline{A}$ ? [les élèves : vecteur A] c'est un vecteur?

Les élèves 12 : NUL !

L'enseignant 13 : I vers A et I vers B regarDEZ bien / I vers A c'est le sens de I vers A / I vers B c'est le sens de I vers B / est c'QUE; ces deux vecteurs sont de même sens?

Les élèves $14:$ NON ; /

L'enseignant 15 : est-c'que ces deux vecteurs sont de même direction? // OUI pourQUOI? parce QUE ces deux vecTEURS sont sur une; même? $\underline{\text { drOITE }}$ [les élèves : drOITE !] est-ce QUE ces deux vecteurs sont de même longueur? Les élèves $16:$ OUI!

Comme pour le cours de français à l'ESCA les interactions verticales ne sont pas individualisées, elles réactivent les connaissances des élèves et leur permettent de mémoriser en français et elles permettent à l'enseignant de s'assurer de leur attention. Les élèves récitent ensemble une définition (tour de parole 6), répètent un terme connu (tours de parole 7 et 15) et répondent très brièvement, et toujours collectivement, aux questions (tours de parole 14 et 16). Le discours de l'enseignant comporte des explications en français dont les grandes lignes sont écrites au tableau. La langue française sera utilisée (bien que l'enseignant ait également recours au malgache) tout au long du cours. Cependant, l'emploi oral du français en classe ne témoigne pas des caractéristiques discursives habituellement attachées à ce canal de communication : les répétitions de l'enseignant s'appuient sur le texte du tableau et ne comportent pas de reformulation, il n'y a pas de véritable échange entre l'enseignant et les élèves qui répondent collectivement et de façon très ritualisée. Bien que le discours de l'enseignant comporte une marque de feedback verbal (tour de parole 11 : A d'accord ») l'aspect interpersonnel de la langue n'est pas saillant et son discours s'apparente au monologue. Dans ce cadre on peut difficilement imaginer un collégien prendre la parole en français de son initiative. 


\section{Conclusion}

Les fonctions des emplois du malgache en classe sont liées au type d'établissement. En effet, dans les collèges publics le malgache occupe sans conteste la place de médium officieux alors que dans les établissements privés c'est le français, qui assure également les fonctions d'explication et d'évaluation.

Soulignons que dans tout type d'établissement, le français permet l'usage d'une terminologie commune relative à la matière enseignée. En ce sens, il remplit la fonction d'objectivisation du savoir. Les déclarations des collégiens et des enseignants dénotent de représentations sociales de références ambigües, à l'image des tensions entre les prescriptions ministérielles, la valorisation sociale $d u$ français et les besoins pragmatiques ressentis par les enseignants.

Enfin, toutes nos observations tendent à prouver que les usages des langues au collège reflètent les rapports diglossiques (et par conséquent les rapports sociaux inégalitaires des locuteurs), à l'œuvre à Madagascar. La systématisation de l'emploi d'une langue ou l'autre dans la communication pédagogique remplit une fonction de sélection, l'école reproduisant les inégalités sociales à travers le système de transmission des savoirs (Bernstein, 1975). La compréhension approfondie des facteurs pesant sur les trajectoires scolaires des collégiens malgaches nécessite pourtant l'examen des multiples relations entre le langage, le savoir et les pratiques de transmission. Une étude élargie que nous avons menée sur ce sujet, souligne des dynamiques d'apprentissages différenciées entre les deux villes et les établissements, ce qui soulève la question des inégalités scolaires et de leur maintien.

\section{BIBLIOGRAPHIE}

Bavoux, C. (2000). Le français de Madagascar : contribution à un inventaire des particularités lexicales. Louvain : Duclot.

Bernstein, B. (1975). Classe et pédagogie : visibles et invisibles. Paris : CERI-OCDE.

Blanchet, P. (2012). La linguistique de terrain, méthode et théorie. Rennes : Presses Universitaires de Rennes.

Bloch, D. (2015). Langues, pratiques de transmission et dynamiques d'apprentissage au collège à Madagascar, Thèse de doctorat. Rouen, Université de Rouen.

Bourdieu, P. (1977). «Sur le pouvoir symbolique ». Annales, Économies, Sociétés, Civilisations, $32^{\text {ième }}$ année, p. 405-411.

Calvet, L.-J. (1999). Pour une écologie des langues du monde. Paris : Plon.

Chiss, J.-L. (2005). « Enseigner et apprendre en français. Des langues de l'école aux discours didactiques ». Le français dans le monde Recherches et applications. Paris : Clé-International, p. 59-64. 
Coste, D., Moore, D. \& Zarate, G. (1997). Compétence plurilingue et pluriculturelle. Strasbourg :

Conseil de l'Europe.

Da Silveira, Y. \& Hamers, J. (1990). « Scolarisation et bilingualité en contexte africain : un défi ? ». Langage et société, 52, p. 23-58. Disponible à : https://web/revues/home/prescript/article/ lsoc_0181-4095_1990_num_52_1_2480.

Dumont, D., Rakotozanany, E. \& Ratsimbazafy, A. (1995). « L'IREM de Madagascar et le problème de la langue d'enseignement ». Repères, 18.

Eagleton, T. (1991). Ideology. An introduction. London : Verso.

Gumperz, J. J. (1989). Sociolinguistique interactionnelle - Une approche interprétative. Paris : L'Harmattan.

Lahire, B. (1993). Culture écrite et inégalités scolaires. Sociologie de "l'échec scolaire ». Lyon : Presses Universitaires de Lyon.

Mackey, W. F. (2000). « Prolégomènes à l'analyse de la dynamique des langues ». DiversCité Langues. Disponible à : http://www.teluq.uquebec.ca/diversite.

Motchane, J.-L. (1990). « Chercher inventer innover dans sa langue ». Dans Cassen, B. (dir). Quelles langues pour la Science? Paris : La découverte.

Py, B. (2004). « Pour une approche linguistique des représentations sociales ». Langages, 154.

Raison-Jourde, F. (1991). Bible et pouvoir à Madagascar au XIXème siècle : invention d'une identité chrétienne et construction de l'État. Paris : Karthala.

Rajemisa-Raolison, R. (1972). Grammaire malgache. Fianarantsoa : Librairie Ambozontany

Razafindratsimba, Dominique, T. (2009). « Le malgache, le français et le variaminanana pratiques plurilingues et représentations linguistiques à Madagasca ", Le français en Afrique. pp. 135-152.

\section{NOTES}

1. Cette variété est nommée le kizanatany, ou plus communément le majungais.

2. Py ne s'intéresse pas, dans le cadre de cette étude, au discours métalinguistique, caractéristique d'une activité réflexive du locuteur sur le langage, mais aux représentations sociales traitant le langage comme « un objet social à interpréter.» (2004, p. 7).

3. Ces deux questions étaient éloignées l'une de l'autre (question $n^{\circ} 4$ et question $n^{\circ} 11$ ) dans le questionnaire afin de minimiser l'influence que la première question pouvait avoir sur la seconde 4. La question concernait le code utilisé selon l'interlocuteur : «Quelle(s) langue(s) parles-tu :avec les professeurs? - avec les parents ? - avec les frères et sœurs ? - avec les amis ?». La question était ouverte et les élèves étaient invités à donner plusieurs réponses. Cependant, l'ensemble des collégiens interrogés n'ont répondu que par « français » ou « malgache ».

5. Nous supposons donc que les élèves ont compris la question de la manière suivante : «Dans quelle langue te parle le professeur?». Nous estimons ainsi que ces graphiques décrivent les échanges enseignant-élèves.

6. Le souvenir de l'échec de la malgachisation de l'enseignement s'accompagne d'une représentation positive de l'enseignement intégralement dispensé en français, langue de mobilité et de promotion sociale.

7. La question était : «Tu préfères quand le professeur explique en quelle langue ?» La réponse était ouverte. 
8. F. Raison-Jourde souligne que les revues religieuses, premiers supports véhiculant une langue malgache pure au XIXème siècle, vont influencer les pratiques linguistiques des élèves sortis des grandes écoles, ces derniers se forçant à perpétuer le purisme élitiste et à renvoyer cette vision idéale de la langue.

9. Le caractère performatif des idéologies a été mentionné par plusieurs théoriciens notamment ceux défendant une approche fonctionnelle du discours idéologique. Selon Eagleton, il existe une sorte «d'état glissant ou de duplicité enfermé dans le langage idéologique » qui permet au discours idéologique d'accomplir certains actes ou fonctions en même temps qu'il prétend constater ou décrire des faits (Eagleton, 1991, p. 19).

10. La lettre D désigne l'enquêteur.

11. La pragmatique malgache est fortement orientée vers la recherche de la préservation de la face de l'interlocuteur.

12. C.E.G. : Collège d'Enseignement Général. Il s'agit d'un établissement public.

13. Soulignons que depuis la malgachisation de l'enseignement, l'idée que les cours d'histoire doivent se faire en langue nationale a perduré. Au début des années 90 , la politique de retour au français précise, par la note circulaire du 7 juillet 1992, que la langue nationale reste médium de l'enseignement pour les matières véhiculant les valeurs malgaches, dont l'histoire (de Madagascar) fait partie.

14. Chiss définit la langue d'enseignement comme celle servant à « la mise en forme des savoirs (contenus, manuels, etc....) » et la langue des apprentissages comme « les formes linguistiques et rhétoriques dans lesquelles les tâches scolaires sont codifiées (explications, consignes, évaluations, etc....). » (Chiss, 2005).

\section{RÉSUMÉS}

Le présent article propose une étude des formes et des fonctions des langues présentes dans la communication pédagogique dans des classes de collèges de deux villes de Madagascar. Selon une analyse croisée de trois corpus de natures différentes recueillis auprès des enseignants du secondaire et de leurs élèves, nous présentons une étude comparative des représentations linguistiques, des pratiques langagières et des modalités de transmission des connaissances dans des établissements d'Antananarivo et Majunga. L'hétérogénéité des pratiques et des représentations linguistiques et les enjeux inhérents révélés par l'analyse, dénotent d'une dynamique de maintien des inégalités scolaires, miroirs des disparités sociales à Madagascar.

In this paper we study the features and functions of educational languages in high schools of two Madagascar's cities. From a cross-analysis of three different data sets collected with school teachers and their students, we present a comparative study of linguistic representations of language practices and knowledge transfer methods in higher education establishments of Antananarivo and Mahajanga. The revealed heterogeneity of linguistic uses and believes/ preconceived as well as their intrinsic challenges, denote a dynamic of educational inequality maintenance mirror Madagascar's social disparities. 
INDEX

Mots-clés : pratiques langagières, inégalités scolaires, alternance codique, langue

d'enseignement

Keywords : language practices, educational inequalities, code-switching, language of instruction Thèmes : Articles pédagogiques

\section{AUTEUR}

\section{DAPHNÉ BLOCH}

Université de Rouen - Dylis - EA 7474

Daphné Bloch est docteure en Sciences du langage, professeure de Français Langue Etrangère et de Lettres modernes. Elle a enseigné en Afrique Subsaharienne et dans l'Océan Indien. Sa thèse a analysé l'impact des pratiques de transmission des savoirs et celui des pratiques langagières sur les trajectoires scolaires, dans des classes de différents collèges à Madagascar.

daphnebloch80[at]gmail.com 